\title{
Low-Cost 3D-Printed Reactionware for the Determination of Fatty Acid Content in Edible Oils using a Base-Catalyzed Transesterification Method in Continuous Flow
}

\author{
A. du Preez ${ }^{1} \cdot$ R. Meijboom ${ }^{1} \cdot$ E. Smit ${ }^{1}$ (I)
}

Received: 14 August 2021 / Accepted: 21 February 2022 / Published online: 8 March 2022

(c) The Author(s) 2022

\begin{abstract}
A low-cost flow system was designed, manufactured, and tested to perform automated base-catalyzed transesterification of triacylglycerols to determine the fatty acid content in edible oils. In combination with traditional gas chromatographic analysis (GC-FID), this approach provides a semi-automated process that requires minimal manual intervention. The main flow system components, namely syringe pumps, connectors (i.e., flangeless fittings), and reactors, were manufactured using 3D-printing technology, specifically fused deposition modeling (FDM). By fine-tuning 3D-printer settings, high-quality leak-tight fittings with standard threading were manufactured in polypropylene (PP), which reduced the overall cost of the flow system significantly. Due to the enhanced reactivity in flow, lower catalyst concentrations $(\leq 1.5 \mathrm{wt} . \%)$ were needed compared to traditional batch reactions $(5 \mathrm{wt} . \%)$. The suitability of the automated flow method was determined by comparing results with the certified fatty acid content in sunflower seed oil from Helianthus annuus. Acceptable levels of accuracy (relative errors $<5 \%$ ) and precision (RSD values $\leq 0.02 \%$ ) were achieved. The mostly 3D-printed flow system was successfully used to determine the fatty acid content of sunflower and other commercial edible oils, namely avocado oil, canola oil, extra virgin olive oil, and a canola and olive oil blend. Linoleic acid (C18:2) was the major component in sunflower oil, whereas all other oils consisted mainly of oleic acid (C18:1). The fatty acid content of the edible oils was comparable to certified and literature values.
\end{abstract}

Keywords 3D-printing $\cdot$ Flow chemistry $\cdot$ Base-catalyzed transesterification $\cdot$ Edible oil $\cdot$ Fatty acid methyl ester

\section{Introduction}

Analytes are often derivatized to increase volatility and reduce polarity, making them more amenable to gas chromatographic analysis. In the food industry, the determination of the fatty acid content of edible oils by the derivatization of triacylglycerol molecules to glycerol and its corresponding three fatty acids (Tammekivi et al. 2019) is common practice (de Koning et al. 2001). In general, edible oils consist primarily of palmitic (C16:0), stearic (C18:0), oleic (C18:1), linoleic (C18:2), and linolenic (C18:3) acids. These five major fatty acids determine not only the physical and

E. Smit

esmit@uj.ac.za

1 Research Centre for Synthesis and Catalysis, Department of Chemical Sciences, University of Johannesburg, PO Box 524, Auckland Park, Johannesburg, South Africa chemical properties of the edible oils but influence their nutritional value as well (Tammekivi et al. 2019).

Specialized commercial instruments (e.g., high-end autosamplers) can perform simple reactions automatically and inject the final reaction mixture directly into the gas chromatograph (GC) (de Koning et al. 2001). However, the cost of these fully automated systems does not always outweigh their benefits. In many instances, analytes are derivatized manually (i.e., offline), and many methods have been developed to make this process more reliable and reproducible (Tammekivi et al. 2019).

An alternative approach would be to perform reactions in flow. Reactions in flow have several key advantages compared to conventional batch procedures. These advantages include rapid mixing, efficient heat and mass transfer rates, and faster and safer reactions (Ötvös et al. 2012; Akwi and Watts 2018; Sharma et al. 2018). To the best of our knowledge, the benefits of continuous flow systems have not been leveraged for fatty acid content analysis from 
triacylglycerols in edible oils. The possibility of using flow chemistry for manufacturing biodiesel through transesterification has been illustrated (Guan et al. 2009). In 2012, Duong and Roper (Duong and Roper 2012) developed a microfluidic chip using conventional photolithography and wet etching techniques, specifically for the derivatization of free fatty acids with methanolic- $\mathrm{HCl}$, to produce the corresponding fatty acid methyl esters. However, the high fabrication costs associated with microreactors (Mason et al. 2007) in conjunction with exorbitantly priced commercial flow equipment (Bannock et al. 2014; Akwi and Watts 2018) has led to the development of low-cost reactionware for flow chemistry purposes. These low-cost flow systems mainly consist of open-source syringe pumps (Wijnen et al. 2014; Cubberley and Hess 2017; Neumaier et al. 2019; Booeshaghi et al. 2019) and various flow components that have been fabricated by using additive manufacturing in some way (Kitson et al. 2012; Dragone et al. 2013; Bishop et al. 2015; Neumaier et al. 2019; Price et al. 2020; Vangunten et al. 2020; Harding et al. 2020).

Polypropylene (PP) is the preferred material for the fabrication of reactionware because it has a thermostability of up to $150{ }^{\circ} \mathrm{C}$ (Dragone et al. 2013) and is inert in a wide range of solvents and chemicals; however, it is challenging to $3 \mathrm{D}$ print (Rao et al. 2017). Polylactic acid (PLA) has limited chemical resistivity and can only be used in temperatures ranging between 60 and $66{ }^{\circ} \mathrm{C}$ (Dragone et al. 2013).

A recent review has highlighted the use of 3D-printed and other open-source hardware in separation science (Davis et al. 2021). The work presented here contributes to these aspects of separation science by showcasing the development of a low-cost, mostly 3D-printed flow system for automated sample derivatization prior to GC analysis. The aim was to produce a fully open-source system, which can be manufactured in-house, even with limited resources. To support this, the open-source Poseidon syringe pump system (Booeshaghi et al. 2019) was employed to deliver fluids. Only PP was used as printing material for all fluidic connectors and devices to avoid the need for multimaterial 3D printers. The utility of the developed system was illustrated by using it to perform base-catalyzed transesterification of edible oils for fatty acid content determination.

\section{Experimental}

\section{Chemicals and Materials}

Analytical standards of fatty acid methyl esters (FAMEs), namely methyl palmitate (C16:0), methyl stearate (C18:0 FAME, $\geq 99.0 \%$ ), methyl oleate (C18:1cis FAME, $\geq 99.0 \%$ ), methyl linoleate (C18:2 FAME, $\geq 98 \%$ ), and methyl linolenate (C18:3 FAME, $\geq 98.5 \%$ ) were purchased from Merck.
Additionally, sunflower seed oil from Helianthus annuиs (tested according to the European Pharmacopoeia, Ph. Eur.), methyl nonanoate (C9:0 FAME, $\geq 99.8 \%$ ), 1-tetradecene ( $\geq 99.8 \%$ ), glycerol trilaurate (trilaurin, $\geq 99 \%$ ), 1,4-dioxane ( $\geq 99 \%)$, sodium citrate dihydrate ( $\geq 99 \%$ ), sodium methoxide solution ( $25 \mathrm{wt}$. \% in methanol), heptane $(99 \%)$, and methanol $(\geq 99.9 \%)$ were purchased from Merck. Edible oils, namely avocado oil (AO), canola oil (CO), extra virgin olive oil (EVOO), sunflower seed oil (SO), as well as a blend of canola and olive oil (C\&OO), were bought from a local supermarket. Supelco tetrafluoroethylene (TFE) tubing $\left(1.58 \mathrm{~mm}\right.$ OD $\times 0.8 \mathrm{~mm}$ ID) and flangeless ferrules $\left(1 / 16^{\prime \prime}\right)$ were purchased from Merck. RS PRO clear polypropylene (PP) filament (1.75 mm diameter) was purchased from RS Components. All chemicals, reagents, and materials were used as received.

\section{Design, Modification, and Manufacturing of Flow System Components}

The flow system components were designed or modified using Fusion 360, a cloud-based computer-aided design (CAD) program that offers a free educational license for students. These designs will be made freely available via Figshare (Du Preez et al. 2022). A Prusa i3 MK3S and Creality CR20 Pro 3D printer were used to manufacture the designed components using transparent PP as fabrication material. PrusaSlicer and Creality Slicer 1.2.3 were used to customize print settings and convert the stereolithography (STL) file to a computer numerical control $(\mathrm{CNC})$ programming language, known as a G-code. A G-code sends commands to the $3 \mathrm{D}$ printer resulting in a printed three-dimensional object (Rossi et al. 2018).

Models for the 3D-printed flangeless fittings kit (Fig. 1) were based on the flangeless fittings kit (Upchurch) purchased from Merck (catalog number 58630). Syringe

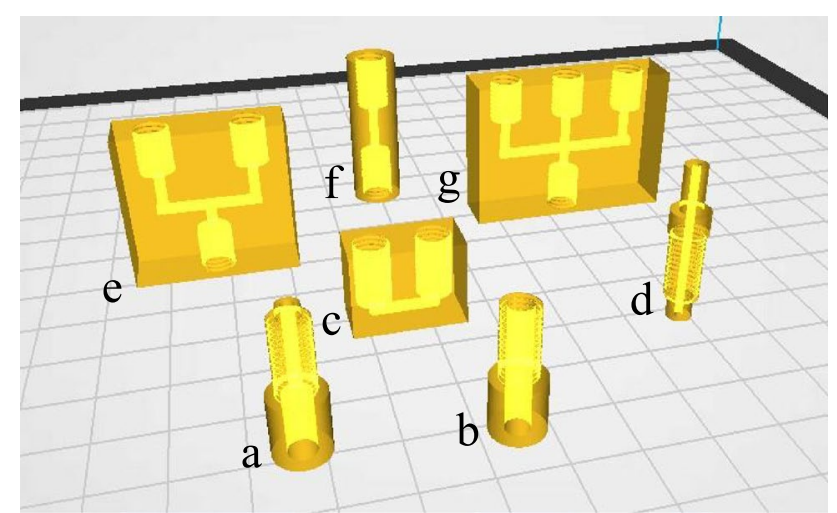

Fig. 1 Designs of 3D-printed components of the flangeless fittings kit include a syringe adapters, $\mathbf{b}$ flangeless nuts, $\mathbf{c}$ alternative unions, $\mathbf{d}$ male Luers, $\mathbf{e}$ T-mixes, $\mathbf{f}$ unions, and $\mathbf{g}$ cross mixers 
adapters, flangeless nuts, male Luers, unions, and mixers (modified t-connectors and crosses) were designed. All mixers were designed with $1.5-\mathrm{mm}$ and $2.0-\mathrm{mm}$ channels which can be readily adjusted. The threaded parts were identical to that of the purchased kit for all designed components, thus making the two kits compatible (1/4"-28 UNF). Additionally, metric unions (M) and metric adapters (1/4"-28 to M6) were also designed similarly to the standard unions in the commercial kit.

Continuous stirred tank reactors (CSTRs) created by Neumaier and co-workers (2019) were modified by replacing the spaces for in-printed screw nuts with threaded access ports that are compatible with the threading of the flangeless nuts (Fig. 2). This modification was crucial because non-metric threaded nuts are scarce in some countries and require unnecessary importation of inexpensive hardware. Moreover, in-printing nuts successfully in PP devices can be challenging. Therefore, fabricating fluidic components with threaded access ports already included by design simplifies the 3D-printing process and makes it more accessible for researchers internationally. The three-inlet CSTR was $36.00 \mathrm{~mm}$ wide and $20.00 \mathrm{~mm}$ high with $2.00-\mathrm{mm}$ channel diameters. The tank diameter was $11.00 \mathrm{~mm}$, and the height from the bottom of the tank to the highest positioned channel was $5.00 \mathrm{~mm}$. Thus, the designed volume of the tank was approximately $0.48 \mathrm{~mL}$.

Figure 3 illustrates the design and modification steps followed for the fabrication of all 3D-printed components. The flangeless fittings were designed and fabricated following route a. The CSTRs were modified and

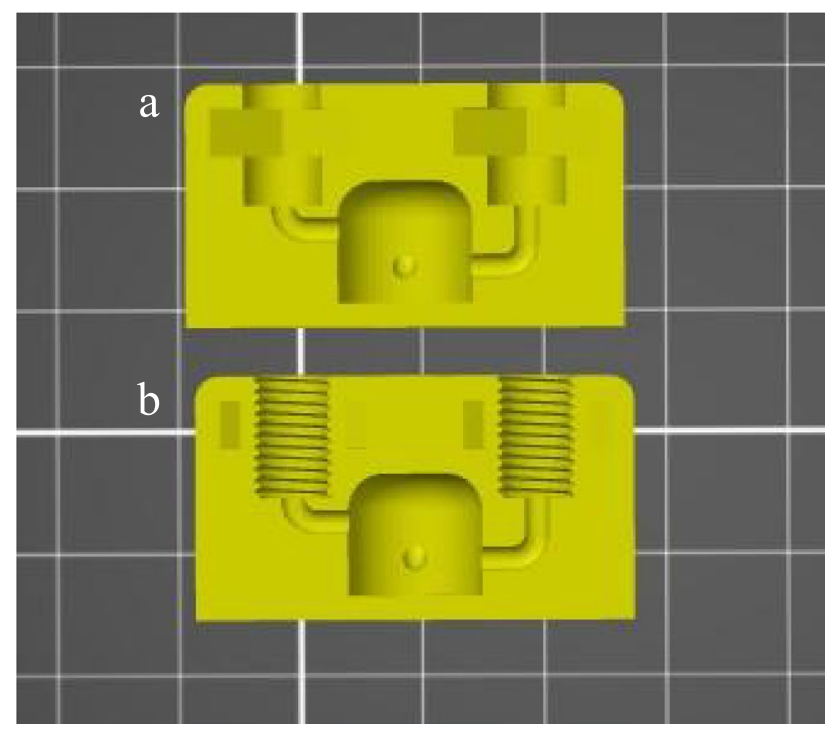

Fig. 2 Cross-sectional view of continuous stirred tank reactors. a Original design from Neumaier et al. (2019) with spaces for inprinted screw nuts, and $\mathbf{b}$ the modified design with threaded access ports

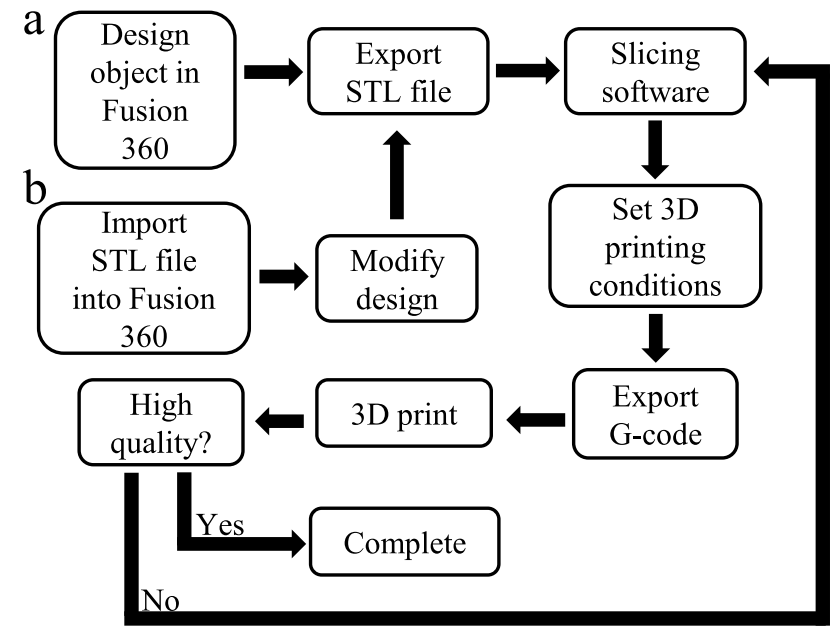

Fig. 3 A flow diagram of the design and modification steps followed in the manufacturing of all flow system components. a Design steps followed for the fabrication of the flangeless fittings and adapters. b The steps followed for the fabrication of modified fluidic devices

manufactured following route $\mathrm{b}$. After printing was completed, the object was inspected. The fittings had to be strong and durable with threaded components that can be inserted and removed without difficulty. Moreover, when connected together and tested with water, it had to be leak-free before the fabrication steps could be considered complete. If any of the before mentioned qualities failed, the slicing software was used to make small changes in the print settings until a high-quality object was obtained.

The optimized printing conditions included a bed temperature of $80{ }^{\circ} \mathrm{C}$ (which was raised to $85{ }^{\circ} \mathrm{C}$ for larger objects to avoid warping). Using an infill of $100 \%$ guaranteed a watertight object, whereas an extruder flow of $105 \%$ provided the necessary tightness of channels (Rao et al. 2017). For both printers, a 0.4-mm nozzle was used together with a layer height of $0.15 \mathrm{~mm}$. No support structures were required except for a brim added to larger objects to improve printer bed adhesion. Furthermore, all components were printed on top of a layer of clear packing tape for strong bed adhesion. The extruder was set to $240{ }^{\circ} \mathrm{C}$ but was systematically reduced to $233^{\circ} \mathrm{C}$ in cases where stringing occurred. This was a crucial step in optimizing the threaded components printed in PP since considerable stringing in printed threaded access ports is troublesome to remove without damaging the threading. In the presence of stringing, it becomes difficult to connect the threaded male and female components. The printing speed also played a crucial role in the quality of the final object; in general, smaller components were printed with slower overall printing speeds. The CSTRs were paused during printing at $46 \%$ to insert a $10 \mathrm{~mm} \times 3.0 \mathrm{~mm}$ magnetic stirrer bar. 
Digital models of the ferrules in the flangeless fittings kit were created; however, it was challenging to fabricate due to its small size. Resin-based printers such as stereolithography (SLA) can be considered for their higher resolution printing capabilities, but the photopolymer materials typically used show poor resistivity to common organic solvents (Rao et al. 2017; Neumaier et al. 2019). Based on this, commercial flangeless ferrules were used in the flow system presented here. The 3D-printed flangeless nuts are compatible with both types of commercial flangeless ferrules (i.e., 1/16" and $\left.1 / 8^{\prime \prime}\right)$, thus giving versatility in tubing size.

\section{Flow System Configuration}

Four 3D-printed Poseidon syringe pumps (Booeshaghi et al. 2019) were used to carry out the flow reactions for the transesterification of triacylglycerols in edible oils (see supporting information, S1). Figure 4 depicts the configuration of the flow system. All fittings and reactors were 3D-printed and used together with commercial Luer slip syringes, TFE tubing, and flangeless ferrules.

A solution containing $1 \mathrm{mg} / \mathrm{mL}$ of each internal standard (trilaurin, C9:0 FAME, and 1-tetradecene) in 1,4-dioxane was prepared. For each of the oils investigated, $500 \mathrm{mg}$ of oil was dissolved in $50 \mathrm{~mL}$ of this solution. All flow reactions were carried out at room temperature. The first CSTR (Fig. 4c) was used to mix the oil-dioxane mixture and the methoxide solution efficiently. The sodium methoxide (in methanol) acts as the base-catalyst, while the 1,4-dioxane acts as a mediator solvent that creates a single-phase system. Heptane was added to the second CSTR (Fig. 4e) for the extraction of the FAMEs. Again, the single-phase system was maintained by mixing. Upon addition of an aqueous sodium citrate solution $(0.15 \mathrm{~g} / \mathrm{mL})$, the reaction was

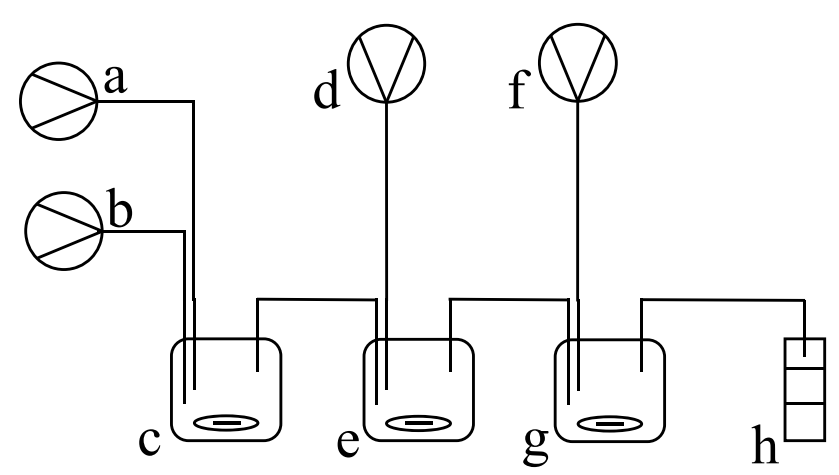

Fig. 4 Schematic diagram of the flow system used for the transesterification of triacylglycerols in edible oils, using a base-catalyzed method at ambient temperature. All three CSTRs contained a stirrer bar and were rapidly stirred using magnetic stirrers. a Dioxane containing the oil and internal standards. b Sodium methoxide. c CSTR 1. d Heptane. e CSTR 2. f Sodium citrate. g CSTR 3. h Sample collection quenched (Fig. 4g), and the organic and aqueous phases separated in the sample collection vial (Fig. 4h). The top layer (organic phase) was transferred to a GC vial for further analysis. For each experiment, three samples were collected at 5-min intervals to evaluate the stability of the flow reaction over time.

Preliminary experiments with fluidic devices based on channel reactors were performed. However, a significant amount of saponification occurred, resulting in solid formation and consequently blocked channels. The use of these chip-based fluidic devices for transesterification will be investigated in more detail in future.

\section{Gas Chromatographic Analysis}

All collected fractions were analyzed with a Perkin Elmer Clarus 580 gas chromatograph using a Supelcowax ${ }^{\circledR} 10$ capillary column $(30 \mathrm{~m} \times 250 \mu \mathrm{m} \times 0.25 \mu \mathrm{m})$. A $1.00-\mu \mathrm{L}$ sample was injected, and the inlet temperature was $250{ }^{\circ} \mathrm{C}$. Helium was used as the carrier gas with a $1.00 \mathrm{~mL} / \mathrm{min}$ flow rate and a split ratio of $20: 1$. The GC oven temperature was set to first reach $180{ }^{\circ} \mathrm{C}$ at $25^{\circ} \mathrm{C} / \mathrm{min}$ from $40{ }^{\circ} \mathrm{C}$, followed by an increase in oven temperature to $250{ }^{\circ} \mathrm{C}$ at $15^{\circ} \mathrm{C} / \mathrm{min}$ with a 3-min hold. The oven equilibrium time was extended to $120 \mathrm{~s}$ to minimize peak shifts between runs. The flame ionization detector (FID) was set to $300{ }^{\circ} \mathrm{C}$. The identity of chromatographic peaks was confirmed by comparison with retention times of standards.

\section{Transesterification Reactions}

Three internal standards were incorporated into the flow reaction prior to $\mathrm{GC}$ analysis by including it in the oil-dioxane solution that was injected into the first CSTR. According to literature, the ratio of the C9:0 FAME peak area relative to the 1-tetradecene peak area indicates that saponification has occurred if it is less than 0.67. In addition, if the C12:0 FAME (originating from trilaurin) to 1-tetradecene peak area ratio is less than 0.75 , transesterification was incomplete, or saponification has already occurred (Suter et al. 1997). These ratios were used to optimize the flow reaction conditions to ensure that the transesterification reaction was complete, but at the same time to ensure that the reaction was terminated before saponification could occur. These initial optimization reactions were carried out using the certified sunflower oil.

The flow rate of individual streams entering the flow system, as controlled by the syringe pumps, played an essential role in the extent of transesterification and saponification. Importantly, in cases where the reaction was not sufficiently quenched, solids formed due to saponification, which resulted in blocked flow channels and devices. The optimized flow rate for the oil-dioxane and sodium methoxide 
solutions was $3 \mathrm{~mL} / \mathrm{h}$. The heptane and sodium citrate solutions were introduced at $12 \mathrm{~mL} / \mathrm{h}$ and $9 \mathrm{~mL} / \mathrm{h}$, respectively.

Various catalyst concentrations were considered. Initially, a $5 \mathrm{wt} . \%$ solution of sodium methoxide was used (Suter et al. 1997) and lower concentrations (1, 0.84, and 0.084 wt.\%) were also considered. Based on preliminary results (see supporting information, S2), the $1 \mathrm{wt} . \%$ solution was used in further experiments.

Consequently, the flow reactions were also compared to reactions performed in batch. Batch reactions were performed based on a previously reported method (Suter et al. 1997), but the catalyst concentration was reduced to $1 \mathrm{wt} . \%$ in order to compare results with those obtained from flow reactions. Batch reactions were performed by transferring $100 \mu \mathrm{L}$ each of the dioxane solution (including the internal standards) and the sodium methoxide solution in a $2-\mathrm{mL}$ vial. The mixture was vortexed for $10 \mathrm{~s}$ and allowed to stand for $60 \mathrm{~s} .1 \mathrm{~mL}$ heptane was added and vortexed for $10 \mathrm{~s}$, followed by the addition of $300 \mu \mathrm{L}$ of a $0.15 \mathrm{~g} / \mathrm{mL}$ sodium citrate solution. The solution was vortexed for $10 \mathrm{~s}$, and complete phase separation was observed after $60 \mathrm{~s}$. The organic phase was then transferred to a GC vial for further analysis.

For each of the oils, the transesterification reaction was performed in triplicate in flow. For each reaction, three fractions were collected at 5-min intervals, followed by GC analysis. The relative peak area of each FAME was determined as a percentage of the sum of the areas of all FAME peaks. Solvent peaks, internal standard peaks, and peaks with an area $<0.05 \%$ were excluded from this calculation. This approach is in line with $\mathrm{Ph}$. Eur. guidelines.

\section{Results and Discussions}

\section{Flow System Components}

To illustrate the versatility and cost-saving capability of 3D-printing technology, a flangeless fittings kit was designed and fabricated with PP as printing material. Print settings and object orientation are crucial to obtain leak-free fluidic devices with well-defined channels. The components were orientated so that the threaded parts were printed vertically (along the $z$-axis). In line with previous studies (Price et al. 2020), this resulted in higher quality fittings.

The 1/4"-28 UNF fittings and connectors manufactured in-house were fully compatible with the commercial kit. This compatibility leads to a significant advantage because damaged or misplaced pieces can be easily replaced at lowcost, or the complete self-manufactured kit can be used on its own. Furthermore, Fig. 5 shows that threaded parts were not significantly altered after being used in a flow system. Only minor damage was observed at the end of the threading (Fig. 5b). The PP fittings were reused several times during the course of this study without any problems, but it is important to note that they do not have an infinite lifespan. A cost analysis (see supporting information, S3) showed that approximately $78 \%$ of research funds could be saved by utilizing $3 \mathrm{D}$ printing for self-manufacturing a flangeless fittings kit. These fittings were also printed using polylactic acid (see supporting information, S4) but have limited use in flow reactions since they are not compatible with most organic solvents.

A similar kit was recently produced by Price et al. (2020), where a multimaterial $3 \mathrm{D}$ printer was used to make standard fittings: wetted parts (those in contact with solutions) were made from PP. In contrast, non-wetted parts (specifically threaded parts) were made from PLA. The reason for their approach was based on the physical properties of PP (e.g.,

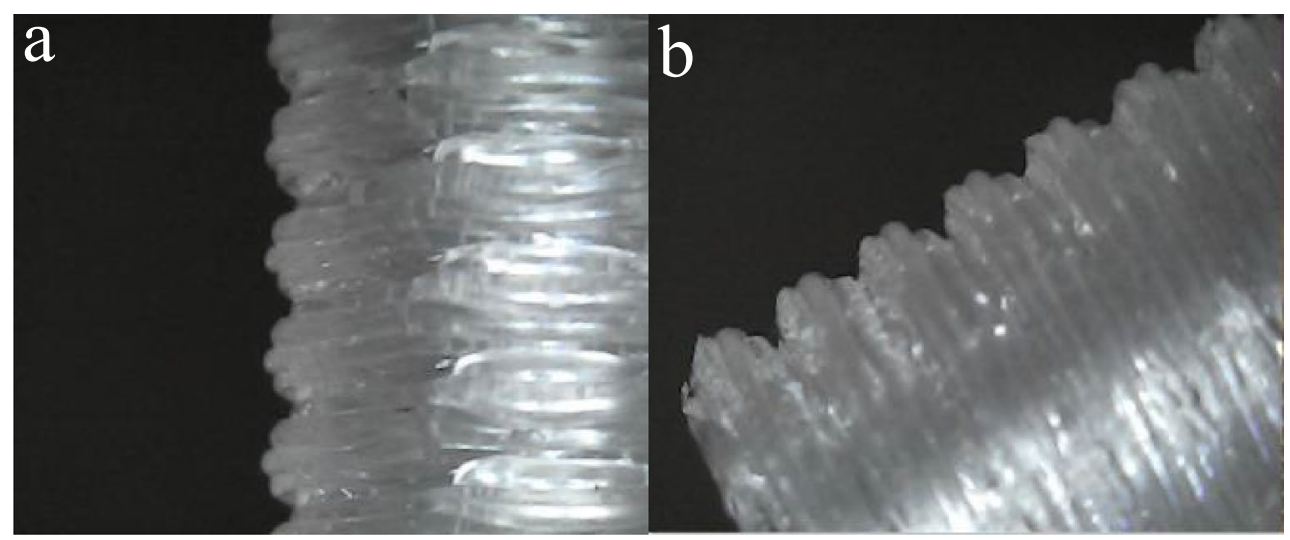

Fig. 5 Threaded parts of 3D-printed PP flangeless nuts, $\mathbf{a}$ before and $\mathbf{b}$ after being used in a flow system 
low flexural modulus), suggesting that it could potentially lead to leaks in a flow system (Price et al. 2020). However, in the work presented here, leak-tight fittings, unions, and mixers were produced with PP by carefully fine-tuning print settings. Although printing with PP is more difficult and troubleshooting can sometimes take longer, the advantage is that a multimaterial $3 \mathrm{D}$ printer is not required to manufacture the components needed to build a flow system.

\section{Flow System Assembly and Operation}

The flow system was set up according to the diagram in Fig. 4 and is shown in Fig. 6. The 3D-printed syringe pumps further contribute to lowering the overall cost of the flow system. All fittings and unions were 3D-printed in PP and were used similarly as commercial fittings. This system is considered to be semi-automated because the fractions are collected and manually transferred to a GC vial, but the actual chemical reaction (in flow) is fully automated. The minimal manual intervention required provides the advantage of minimizing personal errors and potentially improving precision. This methodology is considered safer compared to a batch protocol because limited handling of chemicals was required. Besides, a lower catalyst concentration could be used due to the increased reactivity observed in flow.

\section{Comparison of Reactions in Batch and Flow}

Results obtained for reactions in batch ( $1 \mathrm{wt} . \%$ and $5 \mathrm{wt} . \%$ sodium methoxide) and flow ( $1 \mathrm{wt} . \%$ sodium methoxide) are shown in Fig. 7, where the peak area ratios of internal standards were used to evaluate the extent of transesterification and saponification. No saponification was observed for

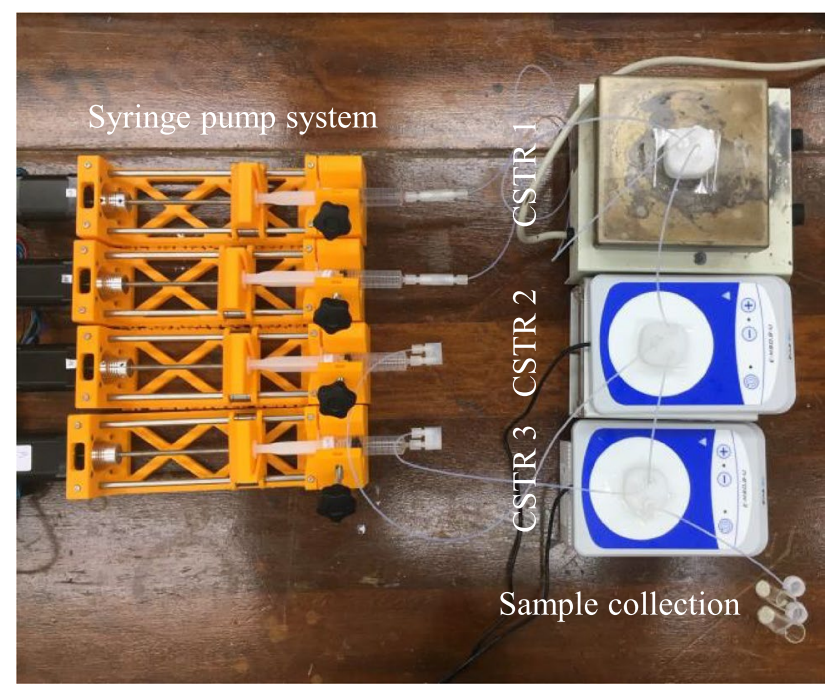

Fig. 6 The setup of the semi-automated 3D-printed flow system for transesterification of edible oils both batch and flow reactions because all C9:0 FAME to 1-tetradecene ratios were above the saponification limit at 0.67 . However, the results indicate that the transesterification reactions performed in batch were incomplete, based on the average C12:0 FAME to 1-tetradecene ratio at 0.71 (Suter et al. 1997). Notably, flow mode outperforms its batch counterpart by having all C12:0 FAME to 1-tetradecene ratios above 0.75 , indicating complete transesterification. This observation can be accounted for due to the reduced reaction time of flow reactions because of efficient mixing and accurate control over reaction parameters in small reactors, which enhances chemical selectivity and improve product yield (Akwi and Watts 2018).

\section{Suitability of the Flow System for Transesterification}

The fatty acid content of sunflower seed oil was determined in triplicate using the mostly 3D-printed continuous flow system, followed by GC-FID analysis. The FA content (expressed as a percentage of total FAMEs observed) was similar for fractions collected at 5, 10, and $15 \mathrm{~min}$ (see supporting information, S5); therefore, only the data for samples collected in the second fraction (10 min) will be considered here. Results were compared to certified values and experimental results obtained from batch reactions (see Table 1). The relative error observed for the reactions performed in batch and flow was similar and less than 5\%, except for C18:0, which had larger relative errors in batch. Since the experiments for the reactions performed in flow yielded results with relative errors below $5 \%$, it was concluded that the 3D-printed flow system could be used to determine FA content with sufficient accuracy. The relative standard deviations are shown in Table 1 also indicate that the precision of both the batch and flow reactions were acceptable. Markedly, the precision of the continuous flow method is comparable with the batch reaction with a higher catalyst concentration.

\section{Fatty Acid Content of Commercial Edible Oils}

The continuous flow system was also used to determine the fatty acid (FA) content of commercial edible oils (Fig. 8 and Table S5). No saponification was observed in any of the reactions. The transesterification was complete for all the oils except the $\mathrm{C} \& \mathrm{OO}$ blend. Consequently, the sodium methoxide concentration was increased slightly to $1.5 \mathrm{wt} . \%$ to drive the reaction to completion.

The FA content of the sunflower and canola oil fall within the established ranges of the Codex Standard for named vegetable oils (Alimentarius 2001a). Additionally, the obtained FA content of sunflower and canola oil is similar to the values reported by Zimmerman and Fick (1973) and Matthaus et al. (2016), respectively. Moreover, the FA content of extra virgin olive oil is in complete agreement with the European 
Fig. 7 A bar chart displaying the difference between the peak area ratios of the internal standards following a batch or flow-mode protocol. All reactions were repeated in triplicate, the height of the bars represents the mean, and the error bars represent the standard deviation

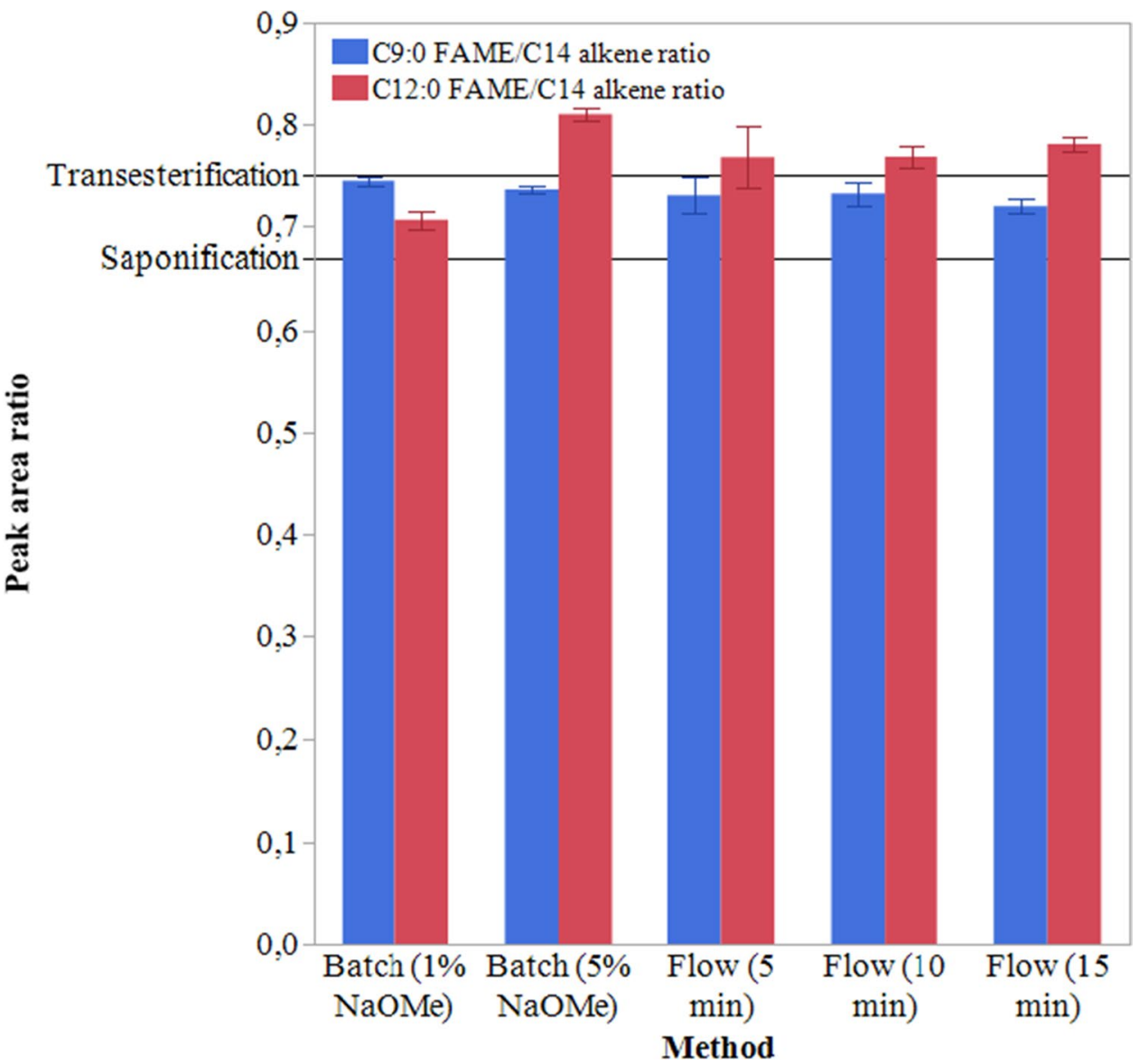

Table 1 Comparison of the certified fatty acid content in sunflower seed oil from Helianthus annuus and values obtained experimentally using an automated flow system and manual batch protocol followed by GC analysis. The experimental results represent the mean value of three replicates, together with the relative standard deviation

\begin{tabular}{|c|c|c|c|c|c|c|c|}
\hline \multirow[t]{2}{*}{ Fatty acid } & \multirow[t]{2}{*}{ Certified values } & \multicolumn{3}{|c|}{ Experimental results $(\%)^{a}$} & \multicolumn{3}{|l|}{ Relative error $(\%)$} \\
\hline & & Flow $(1 \text { wt. } \%)^{b}$ & Batch (1 wt. \%) & Batch (5 wt. \%) & Flow $(1 \text { wt. } \%)^{b}$ & Batch (1 wt. \%) & Batch (5 wt. \%) \\
\hline C16:0 & 6.5 & $6.48 \pm 0.02$ & $6.68 \pm 0.04$ & $6.39 \pm 0.02$ & -0.2 & 2.8 & -1.7 \\
\hline C18:0 & 3.6 & $3.47 \pm 0.01$ & $3.21 \pm 0.06$ & $3.26 \pm 0.04$ & -3.5 & -10.7 & -9.4 \\
\hline C18:1 & 30.6 & $30.39 \pm 0.01$ & $30.29 \pm 0.03$ & $30.78 \pm 0$ & -0.7 & -1.0 & 0.6 \\
\hline C18:2 & 56.8 & $58.5 \pm 0.01$ & $58.07 \pm 0.02$ & $59.4 \pm 0.01$ & 3.0 & 2.2 & 4.6 \\
\hline
\end{tabular}

${ }^{a}$ Expressed as a percentage of total FAMEs observed

${ }^{b}$ Results obtained for the second 5-min fraction

Union Commission Regulation (2016). Since avocado oil has a FA composition similar to olive oil, and there are no internationally established parameters defining the quality of avocado oil, the Codex Standard for olive oil (Alimentarius 2001b) suffices as a reference (Flores et al. 2019).

The observed relative amounts of stearic acid (C18:0), linoleic acid (C18:2), and linolenic acid (C18:3) of the analyzed avocado oil agreed with the established ranges of the Codex Standard for olive oil. The relative amount of palmitic acid (C16:0) slightly exceeded the range of the Codex Standard by approximately $0.1 \%$. However, values for this saturated FA can be as high as 29\% (Abaide et al. 2017). The oleic acid (C18:1) value $(\sim 51 \%)$ was below the Codex Standard minimum of $55 \%$. Tan et al. (2017) reported similar values for this monosaturated FA; however, Santana et al. (2019) and Rydlewski et al. (2020) reported lower oleic acid concentrations of $46 \%$ to $48 \%$, respectively. Methyl palmitoleate (C16:1 FAME) was only tentatively identified and was therefore not included in Fig. 8. This monounsaturated FA (C16:1) presented a value of approximately $10 \%$, which is higher than the established 
Fig. 8 Fatty acid composition of edible oils, based on the percentage of FAMEs observed. The relative amounts represent the average of three replicate fractions collected during the second 5-min interval

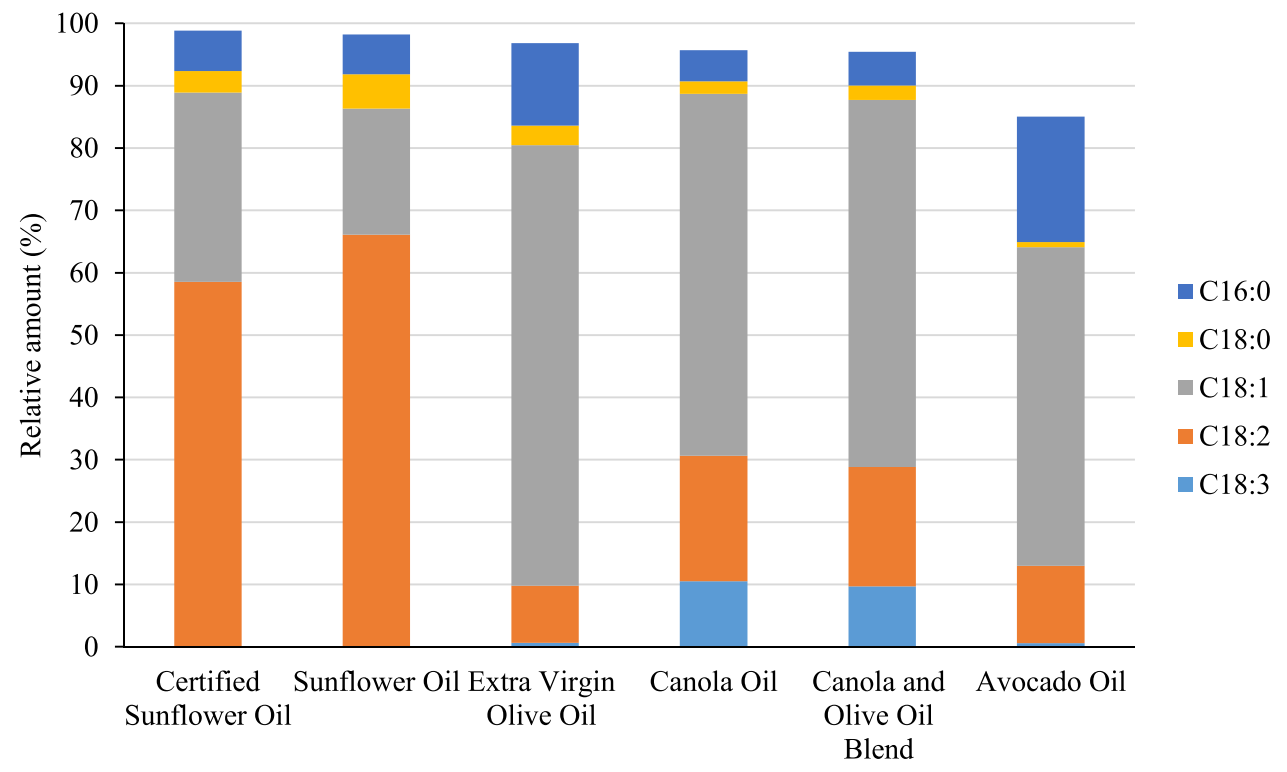

maximum of olive oil (3.5\%). However, similar values have been published for avocado oil elsewhere (Flores et al. 2019). It has been reported that the geographical conditions in which the avocado fruit was grown accounts for variations in FA content (Tan et al. 2017; Flores et al. 2019; Rydlewski et al. 2020).

Sunflower seed oil can be categorized as regular or high-linoleic, mid-oleic, and high-oleic (Alimentarius 2001a; Grompone 2011). The polyunsaturated FA, linoleic acid (C18:2), was the major component in both the certified and commercial SO. Based on this, these oils are of the regular (high-linoleic) type. The commercial SO contained relatively more C18:2 in comparison to the certified oil, $66 \%$ and $58 \%$, respectively. The certified oil contained relatively more oleic acid (C18:1) compared to the commercial version. Both types of SOs had similar amounts of palmitic acid (C16:0) and linolenic acid (C18:3), 6\% and $<0.1 \%$, respectively.

The other commercial oils were high $(>50 \%)$ in oleic acid (C18:1). AO had the highest palmitic acid content $(\sim 19 \%)$, followed by EVOO $(\sim 13 \%)$, SO, and CO, 6\% and $5 \%$, respectively. All oils contain very low levels of $(<1 \%)$ linolenic acid (C18:3), except CO, which contained on average approximately $10 \%$. The FA profile of the C\&OO blend closely resembles that of $\mathrm{CO}$. This suggests that the blend consists mainly of $\mathrm{CO}$, which corresponds with the label on the bottle, which stated that it is a mixture of $90 \% \mathrm{CO}$ and $10 \%$ olive oil.

\section{Conclusion}

The versatility and cost-saving capabilities of 3D-printing technology were demonstrated by reproducing an expensive flangeless fittings kit consisting of syringe adapters, flangeless nuts, male Luers, unions, and mixers that are often used in continuous flow systems. The kit was reproducible in both PLA and PP, although only PP components were used in the flow system presented here. The advantages of the 3D-printed flangeless fittings kit were that misplaced or damaged commercial pieces can be easily replaced or the entire kit can be used independently. The fittings printed in PP could be reused several times but have a finite lifetime. Another disadvantage of this fabrication method was the need to purchase commercial flangeless ferrules, which were too small to 3D print with FDM. The continuous flow system was further expanded by the addition of 3D-printed reactors that were compatible with the 3D-printed fittings. Also, an open-source syringe pump system was used for fluid delivery, lowering the overall cost of the system even more.

A common derivatization technique, namely transesterification, was explored in this work. The mostly 3D-printed flow system was shown to be suitable for performing automated transesterification reactions based on the comparison of experimental results with certified and literature values. The only manual intervention required was preparing reagent mixtures and transferring products to a $\mathrm{GC}$ vial prior to analysis. To illustrate the versatility of the system, various commercial edible oils were also investigated. Although fully automated robotic systems are commercially available and can be used to perform similar derivatization reactions, the semi-automated procedure presented here offers an economically viable alternative to research laboratories. Furthermore, lower catalyst concentrations were required (even at room temperature) compared to traditional reactions due to the enhanced reactivity in flow. Importantly, this flow system has the potential to be adapted for other derivatization reactions in future. 
Supplementary Information The online version contains supplementary material available at https://doi.org/10.1007/s12161-022-02233-2.

Acknowledgements The authors would like to acknowledge the Department of Chemical Sciences at the University of Johannesburg. The authors would like to thank the late Mr. J.A. Hanekom for assistance with building the 3D-printed syringe pumps.

Author contribution E. Smit. and A. du Preez conceived and designed the experiments. A. du Preez designed and manufactured the 3D-printed components and performed the experiments. A. du Preez and E. Smit analyzed the data. R. Meijboom contributed to concepts related to open-source hardware and flow chemistry. E. Smit organized funding. The manuscript was written by A. du Preez and E. Smit. All authors read and approved the final version of the manuscript.

Funding This work is based on the research supported wholly or in part by the National Research Foundation of South Africa (Grant number: 117842)

Data Availability The datasets generated during and/or analyzed during the current study are available from the corresponding author on reasonable request.

\section{Declarations}

Conflict of Interest A Du Preez declares that she has no conflict of interest. R Meijboom declares that he has no conflict of interest. E Smit declares that she has no conflict of interest.

Open Access This article is licensed under a Creative Commons Attribution 4.0 International License, which permits use, sharing, adaptation, distribution and reproduction in any medium or format, as long as you give appropriate credit to the original author(s) and the source, provide a link to the Creative Commons licence, and indicate if changes were made. The images or other third party material in this article are included in the article's Creative Commons licence, unless indicated otherwise in a credit line to the material. If material is not included in the article's Creative Commons licence and your intended use is not permitted by statutory regulation or exceeds the permitted use, you will need to obtain permission directly from the copyright holder. To view a copy of this licence, visit http://creativecommons.org/licenses/by/4.0/.

\section{References}

Abaide ER, Zabot GL, Tres MV et al (2017) Yield, composition, and antioxidant activity of avocado pulp oil extracted by pressurized fluids. Food Bioprod Process I02:289-298. https://doi.org/10. 1016/j.fbp.2017.01.008

Akwi FM, Watts P (2018) Continuous flow chemistry: where are we now? Recent applications, challenges and limitations. Chem Commun 54:13894-13928. https://doi.org/10.1039/c8cc07427e

Alimentarius C (2001a) Codex Standard for named vegetable oils. CXSTAN 210-1999. Codex Aliment 8:11-25

Alimentarius C (2001b) Codex standard for olive oil, virgin and refined, and for refined olive-pomace oil. CODEX-STAN 33-1981 (Rev. 1-1989). Codex Aliment 8:25-39

Bannock JH, Krishnadasan SH, Heeney M, de Mello JC (2014) A gentle introduction to the noble art of flow chemistry. Mater Horiz 1:373-378. https://doi.org/10.1039/c4mh00054d
Bishop GW, Satterwhite JE, Bhakta S et al (2015) 3D-printed fluidic devices for nanoparticle preparation and flow-injection amperometry using integrated prussian blue nanoparticle-modified electrodes. Anal Chem 87:5437-5443. https://doi.org/10.1021/ acs.analchem.5b00903

Booeshaghi AS, Beltrame EdV, Bannon D et al (2019) Principles of open source bioinstrumentation applied to the poseidon syringe pump system. Sci Rep 9:1-8. https://doi.org/10.1038/ s41598-019-48815-9

Cubberley MS, Hess WA (2017) An inexpensive programmable dualsyringe pump for the chemistry laboratory. J Chem Educ 94:7274. https://doi.org/10.1021/acs.jchemed.6b00598

Davis JJ, Foster SW, Grinias JP (2021) Low-cost and opensource strategies for chemical separations. J Chromatogr A 1638:461820. https://doi.org/10.1016/j.chroma.2020.461820

de Koning S, van der Meer B, Alkema G et al (2001) Automated determination of fatty acid methyl ester and cis/trans methyl ester composition of fats and oils. J Chromatogr A 922:391397. https://doi.org/10.1016/S0021-9673(01)00926-8

Dragone V, Sans V, Rosnes MH et al (2013) 3D-printed devices for continuous-flow organic chemistry. Beilstein J Org Chem 9:951-959. https://doi.org/10.3762/bjoc.9.109

Duong CT, Roper MG (2012) A microfluidic device for the automated derivatization of free fatty acids to fatty acid methyl esters. Analyst 137:840-846. https://doi.org/10.1039/C2AN1 5911 B

Flores M, Saravia C, Vergara CE et al (2019) Avocado oil: characteristics, properties, and applications. Molecules 24:1-21. https:// doi.org/10.3390/molecules 24112172

Grompone MA (2011) Sunflower oil, 2nd edn. Wiley-Blackwell, Oxford, UK

Guan G, Kusakabe K, Moriyama K, Sakurai N (2009) Transesterification of sunflower oil with methanol in a microtube reactor. Ind Eng Chem Res 48:1357-1363. https://doi.org/10.1021/ie800852x

Harding MJ, Brady S, O'Connor H et al (2020) 3D printing of PEEK reactors for flow chemistry and continuous chemical processing. React Chem Eng 5:728-735. https://doi.org/10.1039/c9re00408d

Kitson PJ, Rosnes MH, Sans V et al (2012) Configurable 3D-printed millifluidic and microfluidic "lab on a chip" reactionware devices. Lab Chip 12:3267-3271. https://doi.org/10.1039/c2lc40761b

Mason BP, Price KE, Steinbacher JL et al (2007) Greener approaches to organic synthesis using microreactor technology. Chem Rev 107:2300-2318

Matthaus B, Özcan MM, Al Juhaimi F (2016) Some rape/canola seed oils: fatty acid composition and tocopherols. Zeitschrift Für Naturforschung C 71:73-77. https://doi.org/10.1515/znc-2016-0003

Neumaier JM, Madani A, Klein T, Ziegler T (2019) Low-budget 3D-printed equipment for continuous flow reactions. Beilstein $\mathbf{J}$ Org Chem 15:558-566. https://doi.org/10.3762/bjoc.15.50

Ötvös SB, Mándity IM, Fülöp F (2012) Highly efficient 1,4-addition of aldehydes to nitroolefins: organocatalysis in continuous flow by solid-supported peptidic catalysts. Chemsuschem 5:266-269. https://doi.org/10.1002/cssc.201100332

Price AJN, Capel AJ, Lee RJ et al (2021) An open source toolkit for 3D printed fluidics. J Flow Chem 11:37-51. https://doi.org/10. 1007/s41981-020-00117-2

Rao ZX, Patel B, Monaco A et al (2017) 3D-printed polypropylene continuous-flow column reactors: exploration of reactor utility in $\mathrm{S}_{\mathrm{N}} \mathrm{Ar}$ reactions and the synthesis of bicyclic and tetracyclic heterocycles. Eur J Org Chem 2017:6499-6504. https://doi.org/ 10.1002/ejoc.201701111

Rossi S, Puglisi A, Benaglia M (2018) Additive manufacturing technologies: 3D printing in organic synthesis. ChemCatChem 10:15121525. https://doi.org/10.1002/cctc.201701619

Rydlewski AA, Pizzo JS, Manin LP et al (2020) Evaluation of possible fraud in avocado oil-based products from the composition 
of fatty acids by GC-FID and lipid profile by ESI-MS. Chem Pap 74:2799-2812. https://doi.org/10.1007/s11696-020-01119-z

Santana I, Castelo-Branco VN, Guimarães BM et al (2019) Hass avocado (Persea americana Mill.) oil enriched in phenolic compounds and tocopherols by expeller-pressing the unpeeled microwave dried fruit. Food Chem 286:354-361. https://doi.org/10. 1016/j.foodchem.2019.02.014

Sharma MK, Acharya RB, Shukla CA, Kulkarni AA (2018) Assessing the possibilities of designing a unified multistep continuous flow synthesis platform. Beilstein J Org Chem 14:1917-1936. https:// doi.org/10.3762/bjoc.14.166

Suter B, Grob K, Pacciarelli B (1997) Determination of fat content and fatty acid composition through 1-min transesterification in the food sample; principles. Z Lebensm Unters Forsch A 204:252258. https://doi.org/10.1007/s002170050073

Tammekivi E, Vahur S, Kekišev O et al (2019) Comparison of derivatization methods for the quantitative gas chromatographic analysis of oils. Anal Methods 11:3514-3522. https://doi.org/10.1039/ C9AY00954J

Tan CX, Tan SS, Tan ST (2017) Influence of geographical origins on the physicochemical properties of hass avocado oil. J Am Oil Chemi Soc 94:1431-1437. https://doi.org/10.1007/ s11746-017-3042-7
The European Commission (2016) Commission Delegated Regulation (EU) 2016/2095 amending Regulation (EEC) No 2568/91 on the characteristics of olive oil and olive-residue oil and on the relevant methods of analysis. Off J Eur Union L326:1-6

Vangunten MT, Walker UJ, Do HG, Knust KN (2020) 3D-printed microfluidics for hands-on undergraduate laboratory experiments. J Chem Educ 97:178-183. https://doi.org/10.1021/acs.jchemed. $9 b 00620$

Wijnen B, Hunt EJ, Anzalone GC, Pearce JM (2014) Open-source syringe pump library. PLoS One 9:1-8. https://doi.org/10.1371/ journal.pone.0107216

Zimmerman DC, Fick GN (1973) Fatty acid composition of sunflower (Helianthus annuus L.) oil as influenced by seed position. JAOCS, J American Oil Chem Soc 50:273-275. https://doi.org/10.1007/ BF02641352

Du Preez A, Meijboom R, Smit E (2022) 3D-printed Flangeless Fittings. https://doi.org/10.25415/ujhb.19306856.v1

Publisher's Note Springer Nature remains neutral with regard to jurisdictional claims in published maps and institutional affiliations. 